\title{
Diseño y mediación de objetos de aprendizaje
}

Recibido: 8 de junio de 2010
Aceptado: 30 de setiembre de 2010

\begin{abstract}
RESUMEN
La implementación de objetos de aprendizaje en los entornos virtuales ha pasado de ser una iniciativa aislada a toda una corriente de diseño, desarrollo y evaluación de recursos que va más allá de la sola producción para entrar al campo de la cognición y el desarrollo de competencias que subyacen en su propuesta. Por esa razón y dada su importancia en el desarrollo y la calidad de los cursos en línea, este artículo tiene como objetivo primordial definir el concepto de objetos de aprendizaje, discriminar las condiciones técnicas y pedagógicas, así como las implicaciones epistemológicas que deben ser observadas en su proceso de gestión y diseño.
\end{abstract}

\section{PALABRAS CLAVE}

Objetos educacionales, curso en línea, entornos virtuales, diseño instruccional.

\begin{abstract}
The implementation of learning objects in virtual environments has gone from being an isolated initiative to a whole school of design, development and evaluation of resources that goes beyond the mere production to enter the field of cognition and development of skills underlying the proposal. For that reason and because of its importance in the development and quality of online courses, this article aims primarily to define the concept of learning objects, discriminating the technical and pedagogical and epistemological implications that must be observed in the management and design processes.
\end{abstract}

\section{KEY WORDS}

Learning objects, online course, virtual environments, instructional design.

\section{INTRODUCCIÓN}

La integración de los recursos tecnológicos en la educación a distancia se ha fortalecido de manera significativa en los últimos años, como producto de las demandas de la sociedad al buscar nuevas formas de comunicarse, interactuar, negociar y tratar. En este campo, las tecnologías se convierten en medios y herramientas fundamentales para llevar a cabo procesos de mediación, distribución de materiales, interacción, tareas múltiples y lograr los objetivos de aprendizaje (Peters, 2002).

Sin embargo, la sola integración de la tecnología en la educación a distancia no es suficiente, el reto es el desarrollo de procesos de aprendizaje centrados en el estudiante, los cuales fortalezcan los procesos de reflexión y construcción de nuevos conocimientos a partir de su uso (Pensa, 2000). 
Partiendo de estas dos realidades: la integración de las tecnologías de comunicación e información en la educación a distancia y de la necesidad de crear ambientes constructivistas en donde el estudiante sea protagonista de sus procesos de aprendizaje, surge la corriente de creación de objetos de aprendizaje. Concebidos como recursos educativos que permiten no solo la entrega de contenidos, sino también un proceso planificado en el cual se busque el logro de los objetivos a partir de la interacción con el estudiante y la construcción de significados derivados de la comprensión.

El desarrollo de objetos de aprendizaje también ha generado nuevas conceptualizaciones acerca de cómo producir materiales reciclables, flexibles y adaptables a las necesidades y contextos, que faciliten la colaboración entre los educadores (Hodgins, 2000).

Dada la importancia de los objetos de aprendizaje, en este artículo interesa presentar algunas de las principales consideraciones por tomar en cuenta tanto en el proceso de diseño como en la propuesta de mediación.

\section{¿Qué son los objetos de aprendizaje?}

EEl término objeto de aprendizaje se deriva de la noción de "objeto" que propone el campo de la computación y la ingeniería, específicamente de la Programación Orientada a Objetos (POO). Esta manera de programar es así nombrada debido a que el elemento fundamental es el objeto, entendiéndose éste como "cada elemento utilizado en un programa que posee una estructura y forma parte de una organización" (Leestma \& Nyhoff, 1999: 559). En otras palabras, pueden construirse programas y aplicaciones computacionales a partir de objetos que contienen instrucciones y propiedades específicas, que interactúan entre sí como entidades de software independientes.

Individualmente, cada objeto posee una serie de propiedades, atributos y métodos que lo de- finen, por ejemplo: si un texto es un objeto, su color es una propiedad. Son ejemplos de objetos dentro de un programa computacional los documentos que se utilizan, el texto, las imágenes, los vídeos, los botones, entre otros.

El objetivo de la programación por objetos, según Leestma y Nyhoff (1999) es "mejorar la productividad de los programadores facilitándoles reutilizar y extender el software ya existente" (p. 558). Quiere decir entonces que un objeto puede ser integrado en otros programas, dadas su característica de software independiente. Las nociones de extensibilidad y de reusabilidad son claves en el concepto de programación por objetos.

Desde un enfoque pedagógico e instruccional en la literatura, según Wiley (2000) y Gibbons, Nelson y Richards (2000), los objetos de aprendizaje se encuentran referenciados con diferentes nombres, tales como: objetos instruccionales, objetos educacionales, objetos de conocimiento, objetos inteligentes y objetos de datos, entre otros.

Fue el Learning Technology Standards Committee (LTSC) quien seleccionó, según Wiley (2000), el concepto de objeto de aprendizaje, posiblemente retomado de la caracterización que en 1994 usó Wayne Hodgins al referirse a pequeños componentes instruccionales. En ese momento, Hodgins conceptualizó los objetos de aprendizaje como entidades digitales y no digitales; los ejemplos que señaló como tales fueron: contenidos multimedia, contenido instruccional, software y herramientas instruccionales, personas, organizaciones y eventos que dan soporte al proceso instruccional. Sin embargo, para Wiley (2000), así como para algunos grupos específicos de profesionales, la definición que adoptó el LTSC es extremadamente amplia y si bien la definición ha cambiado, el término "objeto de aprendizaje" se conserva.

Wiley (2000) los define como pequeñas entidades digitales (en relación con el tamaño de un curso entero) que crean los diseñadores ins- 
truccionales como componentes de la instrucción y que pueden ser reutilizados en diferentes contextos.

Morales y Agüera (2002) definen el objeto de aprendizaje como "cualquier entidad digital que puede ser usada o referenciada durante una experiencia de aprendizaje apoyada en tecnología computacional o de telecomunicaciones" (p. 26).

Por su parte, González-Barbone y Anido-Rifon (2008) definen un objeto de aprendizaje como "una información reusable, independiente del medio, usada como un bloque modular para crear contenidos de e-learning" (p. 1635). Obsérvese en esta definición la idea de bloque modular, muchos autores comparan la descripción de objetos de aprendizaje con el juego de LEGO. Esta idea de instancias de conocimiento no es nueva, Álvarez, Espinoza y Prieto (s.f.) citan que desde el año 1991 David Merril, en su Teoría de Transacción Instruccional, hace referencia a unidades (estructuras) de conocimiento que pueden ser ensambladas unas con otras.

Se puede concluir entonces que un objeto de aprendizaje es una instancia digital diseñada y desarrollada con el fin de apoyar e incentivar los procesos formativos, compuesto de uno o más elementos electrónicos, sean estos: de texto, de sonido, de imágenes o de animación.

\section{- Condiciones técnicas de los objetos de aprendizaje}

Para facilitar la rápida adopción de objetos de aprendizaje, el Learning Technology Standars Committee (LTSC) del Institute of Electrical and Electronics Engineers (IEEE) creó, en el año 1996, estándares de tecnología instruccional que aseguran la interoperatividad de tecnologías y de manera específica de los objetos de aprendizaje, se trata del estándar Learning Object Metadata (LOM). Este estándar busca satisfacer las necesidades de que los objetos educativos sean de jerarquía creciente, con estructura granular que permitan la agregación o inserción de nuevos objetos y además posean una cobertura curricular (Gértrudix, Álvarez, Del Valle, Gálvez y Gértrudix, 2007).

De forma paralela, en Europa el proyecto ARIADNE (Alliance of Remote Instructional Authoring and Distribution Networks for Europe) con el financiamiento de la Unión Europea y -en Estados Unidos- del Instructional Management Systems (IMS) buscan establecer los estándares que garanticen el desarrollo y el soporte de los objetos y la tecnología instruccional (Wiley, 2000).

Desde el punto de vista técnico, los objetos de aprendizaje deben cumplir con condiciones de formato y de empaquetamiento estándar. El estándar más utilizado es SCORM (Shareable Content Object Reference Model) de ADL (Advanced Distributed Learning). González-Barbone y Anido-Rifon (2008) definen SCORM como una colección de estándares y especificaciones para el empaquetamiento y la secuencia de aprendizaje que permite crear objetos para compartir y reusar.

Gibbons, et ál. (2000) y Wiley (2000) mencionan cuatro cualidades que deben cumplir los objetos de aprendizaje: reusabilidad, generatividad, adaptabilidad y escalabilidad. Las cualidades se detallan a continuación.

Reusabilidad. Supone que el objeto de aprendizaje pueda usarse tantas veces como sea requerido, sin que se le deba hacer cambios significativos y sin perder atributos (Navarro \& Ramírez, 2005).

Generatividad. Gibbons, et al. (2002), indican que un objeto posee este atributo si además de proveer información, a través de los mensajes contenidos en él, es capaz de provocar interacción y generar más conocimiento. "La generatividad se refiere a la habilidad de la instrucción computarizada para crear mensajes instruccionales e interacciones por combinación de los mensajes primitivos y elementos de 
interacción en lugar de almacenar mensajes y lógicas de interacción pre-compuestos" (Gibbons, et al., p. 9). Quiere decir que la generatividad va más allá de una estructura lógica programada a través de reglas en el objeto y busca establecer un diálogo para el desarrollo cognitivo. Suppes (citado por Gibbons, et al., 2000) indica tres niveles de interacción entre los estudiantes y los programas de instrucción que se diseñan: (1) ejercitación y práctica repetitivas; (2) sistemas tutoriales, semejante a la interacción con el profesor en caso de dudas; (3) sistemas de diálogo, que favorezca un verdadero diálogo con la propuesta en el computador y genere conocimiento.

Adaptabilidad. Los objetos de aprendizaje deben brindar diferentes caminos para lograr los objetivos, lo cual puede implicar adaptarse a otros medios y combinarse con recursos de otra índole. Este atributo se refiere, según Gibbons y sus colegas (2000) a la necesidad de una enseñanza más individualizada, adaptable a las necesidades, estilos y capacidades de cada estudiante.

Escalabilidad. Se refiere a la necesidad de crecer y de hacer más eficientes los procesos, reduciendo costos y garantizando calidad. Implica crear modelos que permitan hacer objetos más complejos a partir de unos más simples y de agilizar la producción sin comprometer la calidad. Wayne Hodgins (2000) advierte que los objetos de aprendizaje son unidades o bloques los cuales no deben ser tan pequeños al punto de percibirse como "sub-atómicos" sino que el tamaño estándar que se le otorgue debe permitir literalmente el ensamble en tamaño, forma y función.

Los objetos de aprendizaje, bajo el estándar SCORM deben a la vez cumplir con cuatro principios: reusabilidad, accesibilidad, interoperatividad, durabilidad.

La reusabilidad para SCORM implica el uso en diversos contextos y necesidades, así como en distintas plataformas tecnológicas. Esto es, un objeto puede ser usado de manera transparente en WebCT, en Moodle, en Blackboard, así como en otros sistemas como videoconferencias o sistemas de comunicación como Wimba e Elluminate Live (Álvarez, Espinoza \& Prieto, sf.) sin que esto signifique depreciación en su formato y en su contenido.

La accesibilidad, para Zapata (2005), significa que "las herramientas y productos que sigan estos estándares permiten un seguimiento del comportamiento y del historial de aprendizaje y de índole académico de los alumnos" (p. 12).

La interoperabilidad implica que además de operar en forma óptima, un objeto de aprendizaje, debe permitir su uso en cualquier plataforma (Blackboard, WebCT, Dokeos, Moodle, etc.) Por ello, se dice que debe caracterizarse por asentir la interoperabilidad, es decir, el poder trasladar el recurso a otras plataformas, con la seguridad de que servirá exacta y correctamente.

Por último, la durabilidad significa que "los productos tecnológicos desarrollados con el estándar eviten la obsolescencia de los materiales" (Zapata, 2005: 12).

\section{- Condiciones pedagógicas de los objetos de aprendizaje}

Desde un enfoque pedagógico, los objetos de aprendizaje son recursos didácticos que apoyan en el proceso de enseñanza y aprendizaje. Sin embargo, para que puedan cumplir tal función deben responder a condiciones que garanticen su significatividad y pertinencia en el marco del curso en el cual se integran.

El principal proceso que debe ser considerado en el desarrollo de objetos de aprendizaje es que ellos comportan un proceso particular de diseño instruccional. Es decir, un objeto de aprendizaje tiene sus objetivos, contenidos específicos por desarrollar, una estrategia metodológica para el logro de sus objetivos y una 
propuesta evaluativa. De este proceso de diseño instruccional particular emergen las siguientes condiciones.

Articulación instruccional. Si bien el objeto de aprendizaje es independiente por definición, debe articularse y ser congruente con el diseño del curso en el cual se integra.

Pertinencia educativa. Los objetos de aprendizaje deben procurar el logro de un concepto, una habilidad, un valor, etc. (García, 2005)

Autosuficiencia de contenidos. Son concretos y sintéticos en la información que se brinda al estudiante. Por su constitución son autosuficiente en su contenido. En otras palabras, de manera independiente y autónoma deben proveer la información necesaria para el logro del objetivo.

Escalabilidad. Es decir, se pueden integrar con otros objetos, secuencias y contextos de aprendizaje. Quiere decir entonces que el objeto puede volverse más complejo al integrarse a otros objetos.

Interactividad. Promueven la actividad del estudiante en el proceso de aprendizaje a través de la oferta de experiencias y estrategias que se contemplan en la construcción del conocimiento.

Flexibilidad. El objeto debe responder a las necesidades de los estudiantes y debe facilitar el acceso a la información en el momento y al ritmo que el estudiante decida.

Retroalimentación oportuna. Contienen actividades de verificación de logros y brinda retroalimentación.

Actualidad. Se deben establecer mecanismos de control que faciliten la renovación de la información. Esto está muy ligado, según García (2005) a la flexibilidad del medio en que se produzca.
- Gestión deambientes deaprendizaje basado en el uso de objetos

Los objetos de aprendizaje no constituyen la totalidad del curso en línea. Son elementos adicionales que responden a objetivos instruccionales específicos y que desde el punto de vista temático han sido seleccionados dadas sus características, sean los niveles de dificultad, la comprensión de procesos, o bien, el puntualizar en algunas competencias, entre otros aspectos.

Por otro lado, no todos los objetos de aprendizaje integrados en un curso deben ser diseñados y construidos por el profesor o por el diseñador instruccional del curso. Dado que la principal característica de los objetos es la reusabilidad y esta se manifiesta en un ambiente de curso, cuando de una manera pensada y planificada se integran objetos de aprendizaje que fueron creados para otros cursos y asignaturas. Esta integración implica una evaluación de su significatividad, pertinencia y calidad. Chan (2002) agrega que también deben responder a la "visualización de diversas problemáticas intervenibles desde distintas disciplinas o prácticas profesionales" (p.g).

Desde esta perspectiva, Chan (2002) exhorta sobre la importancia de considerar en la gestión de objetos de aprendizaje tres posibilidades de representación: el objeto mediático, el objeto de conocimiento y el objeto de aprendizaje. Esta investigadora, citando a Merrill, indica:

"un objeto mediático es un conjunto de bits de texto, gráficos, video o audio. Al identificarse algún valor de este objeto como conocimiento para un sujeto, se le puede considerar objeto de conocimiento. Cuando se integra una estrategia instruccional, al objeto mediático, por haberle atribuido valor como conocimiento, se obtiene un objeto de aprendizaje" (Chan, 2002, p.9).

Entonces, se podría graficar así como se muestra a continuación en la Figura 1: 


\section{OBJETO DE APRENDIZAJE}

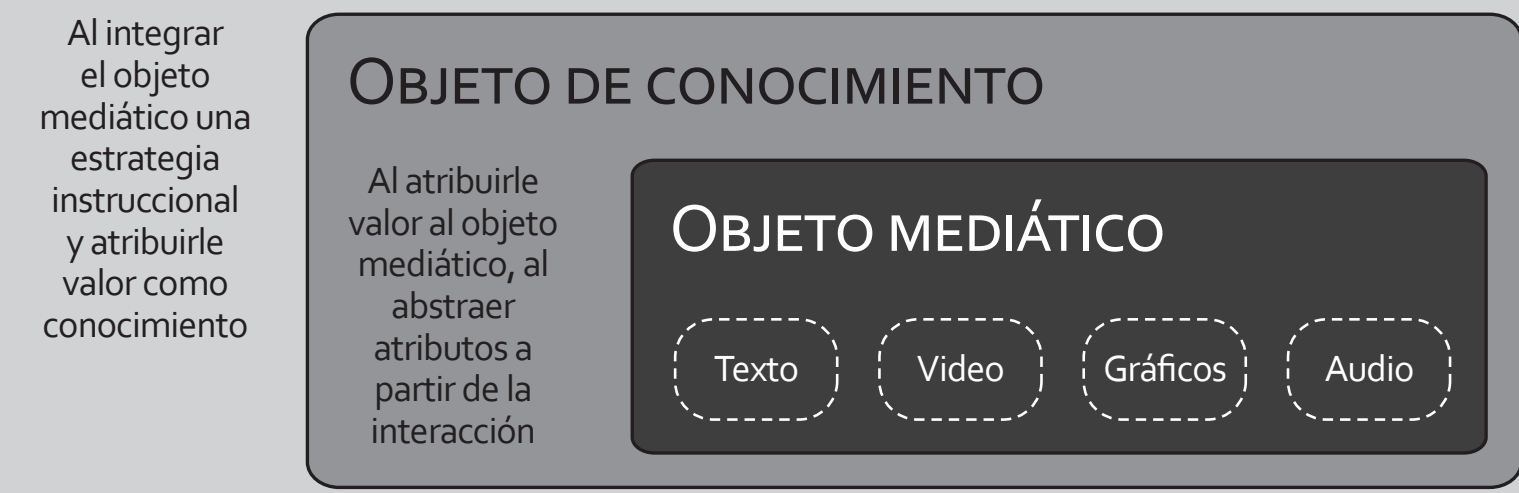

Figura 1. Objetos mediático, de conocimiento y de aprendizaje.

En otras palabras, la información adquiere significado educativo cuando la actividad que se propone sobre ella, genera y da valor al objeto. Este atributo es la generatividad y dentro de él deben ser considerados los niveles de interacción expuestos por Suppes (citado por Gibbons, et ál., 2000) de manera tal que se produzca conocimiento, puesto que la principal intencionalidad del objeto es el aprendizaje.

La interacción que se provoca entre el objeto y los sujetos de aprendizaje debe provenir de dos fuentes:

1. Una serie de instrucciones y estrategias dentro del mismo objeto de aprendizaje que provocan la interactividad con los contenidos y el desarrollo de experiencias para la adquisición de competencias. Desde el punto de vista de la epistemología constructivista esto implica la acción del sujeto con el objeto.

2. Una estrategia instruccional propia de un curso y de una ruta de aprendizaje, externa al objeto que implica una acción docente de acompañamiento y tutoría. En otras palabras un proceso de planificación y estructuración del curso, la cual también deriva de un paradigma de aprendizaje que ha permeado el diseño.

\section{- Consideraciones epistemológicas de objetos de aprendizaje}

Todo objeto de aprendizaje por su naturaleza pedagógica requiere de un diseño instruccional para su construcción. Así, se logra contemplar otros elementos de los cuales se hace referencia a continuación.

Promover la reflexión y el pensamiento crítico. Según Navarro y Ramírez (2005), un objeto de aprendizaje debe proveer de espacios de reflexión y pensamiento crítico de una manera integrada, es por ello que los autores tienen como referencia la propuesta de pensamiento complejo de Morín.

La teoría del pensamiento complejo propone ver el conocimiento como un conjunto de redes indivisibles y de complicado manejo, por lo tanto no es viable delimitar una sola vía para la 
construcción de conocimientos (Morín, 2001). En ese sentido, por su condición de interactividad, los docentes pueden promover redes de objetos de aprendizaje y favorecer el pensamiento complejo (Navarro \& Ramírez, 2005).

Potenciar el desarrollo de competencias. Otro elemento a considerar en el uso de los objetos de aprendizaje es el enfoque educativo por competencias que actualmente tiene gran desarrollo tanto en América como en Europa (Tobón, 2007).

El enfoque por competencias se retoma en el campo educativo con dos objetivos muy claros. El primero apunta al desarrollo de una formación menos fragmentada y más articulada con el medio laboral. En segundo lugar, el interés se centró en la tendencia en buscar criterios comunes para la formación de los profesionales, de manera que se rompan las fronteras entre instituciones y países. Ejemplos claros se tienen en Europa con el proyecto entre los países Tunning ${ }^{1}$, el cual fue desarrollado en el continente americano con el nombre de Tunning-América Latina, otras iniciativas como el proyecto $6 \mathrm{X}_{4}$ y los múltiples esfuerzos que hoy día se realizan en América Latina (Calvo \& Salas, 2008).

En cada experiencia educativa desde el enfoque por competencias se pretende el desarrollo de individuos integrales, por lo tanto su formación contempla el desarrollo de conocimientos, habilidades, destrezas y valores sustantivos para su desenvolvimiento como futuros profesionales y en su vida profesional (Navarro \& Ramírez, 2005).

Por sus características de interactividad, flexibilidad y reusabilidad, el trabajo con objetos de aprendizaje permite el desarrollo de elementos específicos de competencias. También se pueden desarrollar experiencias concretas articuladas con el contexto de los estudiantes, a la vez que propiciar actividades de aprendizaje que se orienten a la resolución de problemas, tanto en el contexto local como regional y global (Navarro \& Ramírez, 2005).

Fomentar la construcción del conocimiento. Todos estos elementos se contemplan en el diseño instruccional del objeto de aprendizaje, que de acuerdo con Chan (2002) debe ser de corte constructivista. Según la autora, el desarrollo de una "estrategia de diseño curricular orientada a desarrollar objetos de aprendizaje, parte de la premisa sobre la indisoluble relación entre el sujeto y el objeto para la definición de este último" (Chan, 2002: 8). En otras palabras, Chan propone desde un enfoque epistemológico constructivista, que el estudiante ejecute acciones sobre la información que brindan los objetos de aprendizaje. Continúa esta autora ampliando al respecto "Los objetos se construyen en función de las capacidades de manipulación, procesamiento, intervención y transformación de dichos objetos" (Chan, 2002: 8) de manera que el estudiante puede interactuar con ellos, además que le permiten la construcción de nuevos conocimientos.

Partir de las premisas anteriores conlleva varias implicaciones, según Chan (2002):

" Hay que delimitar un contenido que responda a una necesidad y un objetivo específico.

» Hay que crear un objeto que implica dar oportunidad al estudiante de considerar la realidad.

» Extraer los atributos del objeto a partir del uso de diversos lenguajes y medios, de manera que los contenidos y las actividades se conviertan en objeto mediático.

\section{CONCLUSIONES}

El contexto actual, donde se evidencia un auge importante de la tecnología, remite a la reflexión sobre las prácticas educativas que tradicionalmente se han venido desarrollando. En estas circunstancias, se determina que ante la sociedad contemporánea se requiere de nuevas y variadas estrategias de aprendizaje, orientadas desde enfoques que tiendan a la 
integración de conocimiento y al rompimiento de los espacios de las disciplinas (Navarro \& Ramírez, 2005).

Este nuevo espacio que se ha denominado la sociedad del conocimiento o de la información (Barnett, 2001), remite a una reflexión del modelo educativo a distancia, en el cual se ha trabajado de una manera sumamente planificada y casi con un esquema de tipo industrial (Peters, 2002). Se requiere entonces de la incorporación de herramientas tecnológicas que funcionen como medios alternativos para el desarrollo de los procesos de enseñanza y aprendizaje (Pensa, 2000).

Es así como los objetos de aprendizaje se constituyen en elementos tecnológicos con una orientación didáctica, cuyas cualidades le dan una nueva posibilidad en recursos para ser utilizados por los docentes.

Algunas de las razones que expone Chan (2002: 4) para integrar objetos de aprendizaje en el quehacer educativo son:

» La mayor aceptación de la tecnología como factor de innovación educativa por docentes y estudiantes.

» La presión para actualizar continuamente los contenidos educativos por el acelerado avance tecnológico.

» La constitución de un mercado en el que el conocimiento adquiere valor.

» La consideración de criterios económicos como la relación costo/beneficio en la prestación de los servicios educativos.

Como todo recurso tecnológico, los objetos de aprendizaje son medios de gran utilidad siempre y cuando surjan con fines claros y contemplen los parámetros establecidos, de manera que deben ser elaborados a partir de un diseño instruccional.

Finalmente, ante las demandas actuales del medio social, en donde se favorecen los es- pacios de articulación entre las disciplinas, es recomendable la construcción de objetos de aprendizaje de corte constructivista. De esta manera, por un lado se trasciende de las fronteras de las disciplinas $y$, por otro lado, se favorecer en los estudiantes, las competencias que les permitan un mejor desenvolvimiento en el ámbito laboral.

\section{NOTAS}

1. Tunning América Latina es un proyecto ALFA auspiciado por la Comunidad Europea en el cual participaron: Argentina, Bolivia, Brasil, Chile, Colombia, Costa Rica, Cuba, Ecuador, El Salvador, Guatemala, Honduras, México, Nicaragua, Panamá, Paraguay, Perú, República Dominicana, Uruguay y Venezuela. El objetivo del mismo es lograr entre los países participantes puntos de referencia en común para la armonización de las carreras que en esta etapa fueron seleccionadas: Administración de Empresas, Arquitectura, Derecho, Educación, Enfermería, Física, Geología, Historia, Ingeniería Civil, Matemáticas, Medicina y Química.

\section{REFERENCIAS}

Álvarez L., Espinoza D. \& Prieto M. (sf). Empaquetamiento de Objetos de Aprendizaje bajo el estándar SCORM

Barnett, ( 2001). Los límites de la competencia. El conocimiento, la educación superior y la sociedad. España: Editoral Gedisa.

Calvo, X. \& Salas, N. (2008). Implementación del enfoque de competencias en la Universidad Estatal a Distancia. ¿Desafío u oportunidad? En: XIV Congreso Internacional de Tecnología y Educación a Distancia. UNED. Celebrado en San José, Costa Rica.

Chan, E. (diciembre, 2002). Objetos de aprendizaje, una herramienta para la innovación educativa. Revista Apertura No. 2

García, L. (2005). Objetos de aprendizaje. Características y repositorios. Editorial del BENED, versión digital. 
Gértrudix, M., Álvarez, S., Del Valle, A., Gálvez, M. \& Gértrudix, F. (2007). Acciones de diseño y desarrollo de objetos educativos digitales: programas institucionales. Universidad Oberta de Catalunya. Revista Universidad y Sociedad del Conocimiento 4 (1) (pp-14-25)

Gibbons, A., Nelson, J. \& Richards, R. (2000). The nature and origin of instructional objects. En: Wiley, D. The Instructional Use of Learning Objects: Online Version. Recuperado el22 de marzo del 2010 de http://reusability.org/read/ chapters/gibbons.doc.

Gonzalez-Barnone, V. \& Anido-Rifon, L. (December 2008). Creating the first SCORM object. Computers \& Education 51, Issue 4 (pp.1634-1647).

Hodgins, H. W. (2000). The future of learning objects. En: Wiley, D. (2000). The Instructional Use of Learning Objects: Online Version. Recuperado el 29 de marzo del 2010 de: http:// reusability.org/read/chapters/hodgins.doc

Leestma, S. \& Nyhoff, L. (1999). Programación en Pascal (4ta. Ed.). Madrid, España: Prentice Hall.

Navarro, J. \& Ramírez, L. (2005). Objetos de aprendizaje. Formación de autores con el modelo redes de objetos. México: Universidad de Guadalajara.

Morín, E. (2001). Los siete saberes necesarios para la educación del futuro. Colombia: Editorial Santillana.
Morales R. \& Agüera A. (enero-febrero 2002). Capacitación basada en objetos reusables de aprendizaje. Boletín IIE, Recuperado el 26 de marzo de: http://www.iie.org.mx/2002a/tendencias.pdf\#search $=\% 22$ Morales\%20R.\%20 y\%20Ag\%C3\%BCera\%20A.\%20(2002)\%20 Capacitaci\%C3\%B3n\%2obasada\%2oen\%20 objetos\%2oreusables\%2ode\%2oaprendizaje.\%2oBolet\%C3\%ADn\%2ollE\%2C\%2oenero-febrero\%2C\%202002\%22

Pensa, D. (2000). Producción de materiales educativos: del texto al hipertexto. En Virtual Educa celebrado en Madrid, España.

Peters, O. (2002). La educación a distancia en transición. Nuevas tendencias y retos. México: Universidad de Guadalajara.

Tobón, S. (2007). El enfoque complejo de las competencias y el diseño curricular por ciclos propedéuticos. Revista Acción Pedagógica. Año XVI, 4 (28).

Wiley, D. (2000). Connecting learning objects to instructional design theory: a definition, a metaphor, and a taxonomy. En: Wiley, D. (2000), The instruccional use of learning objects: Online version. Recuperado el 22 de marzo del 2010 de: http://reusability.org/read/chapters/wiley.doc

Zapata, M. (20 de Febrero de 2005). Secuenciación de contenidos y objetos de aprendizaje. Revista de Educación a Distancia, RED. Año IV. Número monográfico II. Murcia, España. 
